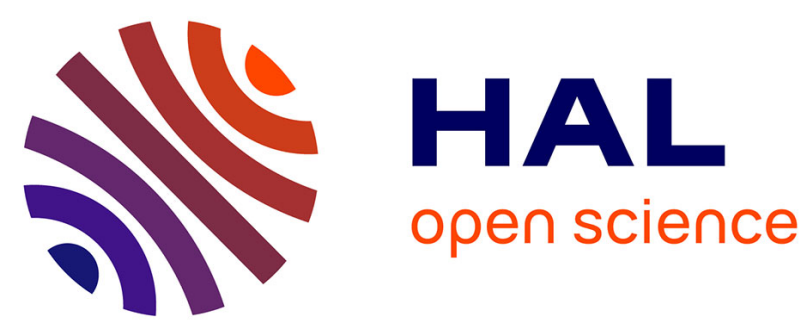

\title{
A New Error Assessment Method in Isogeometric Analysis of 2D Heat Conduction Problems
}

Gang Xu, Bernard Mourrain, Régis Duvigneau, André Galligo

\section{To cite this version:}

Gang Xu, Bernard Mourrain, Régis Duvigneau, André Galligo. A New Error Assessment Method in Isogeometric Analysis of 2D Heat Conduction Problems. Advanced Science Letters, 2012, 10 (1), pp.508-512. 10.1166/asl.2012.3321 . hal-00742955

\section{HAL Id: hal-00742955 \\ https://hal.inria.fr/hal-00742955}

Submitted on 17 Oct 2012

HAL is a multi-disciplinary open access archive for the deposit and dissemination of scientific research documents, whether they are published or not. The documents may come from teaching and research institutions in France or abroad, or from public or private research centers.
L'archive ouverte pluridisciplinaire HAL, est destinée au dépôt et à la diffusion de documents scientifiques de niveau recherche, publiés ou non, émanant des établissements d'enseignement et de recherche français ou étrangers, des laboratoires publics ou privés. 


\title{
A New Error Assessment Method in Isogeometric Analysis of 2D Heat Conduction Problems
}

\author{
Gang $\mathrm{Xu}^{\# 1}$, Bernard Mourrain ${ }^{* 2}$, Régis Duvigneau ${ }^{* 3}$, André Galligo ${ }^{\$ 4}$ \\ ${ }^{\#}$ College of Computer, Hangzhou Dianzi University, Hangzhou, P.R China \\ ${ }^{1}$ gxu@hdu.edu.cn \\ *INRIA Sophia-Antipolis, 2004 Route des Lucioles, 06902 Cedex, France \\ ${ }^{2}$ Bernard.Mourraineinria.fr \\ ${ }^{3}$ Regis.Duvigneaueinria.fr \\ ${ }^{\S}$ University of Nice Sophia-Antipolis, 06108 Nice Cedex 02, France \\ ${ }^{4}$ galligoeunice.fr
}

\begin{abstract}
In this paper, we propose a new error assessment method for isogeometric analysis of $2 \mathrm{D}$ heat conduction problems. The posteriori error estimation is obtained by resolving the isogeometric analysis problem with several $k$-refinement steps. The main feature of the proposed method is that the resulted error estimation surface has a B-spline form, according to the main idea of isogeometric analysis. Though the error estimation method is expensive, it can be used as an error assessment method for isogeometric analysis. Two comparison examples are presented to show the efficiency of the proposed method.
\end{abstract}

Keywords - Isogeometric analysis, posteriori error estimation,

$k$-refinement, heat conduction

\section{INTRODUCTION}

Usually, CAD modeling software relies on splines or NURBS representations, while the CAE software for CAD object uses mesh-based geometric descriptions (structured or unstructured). Therefore, in conventional approaches, several information transfers occur during the design phase, yielding approximations and non-linear transformations that can significantly deteriorate the overall efficiency of the design optimization procedure.

The isogeometric analysis (IGA for short) method proposed by Hughes et al. in [12] can be employed to overcome the gap between CAD and CAE. The approach uses the same type of mathematical representation (spline representation), both for the geometry and for the physical solutions, and thus avoids data transfers between the design and analysis phases. Moreover it reduces the number of parameters needed to describe the geometry, which is of particular interest for shape optimization. This framework allows to compute the analysis solution on the exact geometry (not a discrete geometry), obtain a more accurate solution (high-order approximation), reduce spurious numerical sources of noise that deteriorate convergence. Moreover, NURBS representation is naturally hierarchical and allows to perform refinement operations to improve the analysis result.
Since the concept of isogeometric analysis was proposed, many researchers in the fields of computational mechanical and geometric modeling were involved in this topic. The current work on isogeometric analysis can be classified into three categories: (1) application of IGA to various simulation and analysis problems[2][5][9][11]; (2) application of various modeling tools in geometric computation to IGA [6][10] [7] [14];(3)error estimation, accuracy and efficiency improvement of IGA framework by reparameterization and refinement operations [1][4][3][8][13][15][16].

The topic of this paper belongs to the third field. As far as we know, there are few works on the error estimation method in isogeometric analysis. Bazilevs et al. studied the error estimation for $h$-refined mesh in isogeometric analysis[4]; some estimates for $h-p-k$-refinement in isogeometric analysis are investigated in [3]; Dörfel et al. proposed a posteriori error estimation for local $h$-refinement with $\mathrm{T}$ splines[10]. In [16], an error assessment method is proposed based on $h$-refinement operation. In this paper, we propose a new error assessment method for isogeometric analysis of two-dimensional heat conduction problems, which is obtained by resolving the isogeometric analysis problem with several $k$-refinement steps.

The remainder of the paper is organized as follows. Section II introduces the isogeometric analysis and $k$ refinement for two dimensional heat conduction problems. Section III presents the error assessment method based on $k$ refinement for isogeometric analysis of two dimensional heat conduction problems. Some examples and comparisons with $h$-refinement method are presented in Section IV. Finally, we conclude this paper and outline future works in Section V.

\section{ISOGEOMETRIC ANALYSIS OF HEAT CONDUCTION PROBLEM}

Given a domain $\Omega$ with $\Gamma=\partial \Omega_{D} \cup \partial \Omega_{N}$, for ease of presentation, we consider the two dimensional second order elliptic PDE with homogeneous Dirichlet boundary condition as an illustrative model problem : 


$$
\begin{array}{ll}
\Delta U(\mathbf{x})=f(\mathbf{x}) & \text { in } \Omega \\
U(\mathbf{x})=U_{0}(\mathbf{x}) & \text { on } \partial \Omega
\end{array}
$$

where $\mathbf{X}$ are the Cartesian coordinates, $\Omega$ is a Lipschitz domain with boundary $\partial \Omega, f(\mathbf{x}) \in L^{2}(\Omega): \Omega \mapsto \mathbb{R}$ is a given source term, and $U(\mathbf{x}): \Omega \mapsto \mathbb{R}$ is the unknown solution.

According to a classical variational approach, we seek for a solution $U \in H^{1}(\Omega)$, such as $U(\mathbf{x})=U_{0}(\mathbf{x})$ on $\partial \Omega$ and:

$$
\int_{\Omega} \nabla(\nabla U(\mathbf{x})) \psi(\mathbf{x}) d \Omega=\int_{\Omega} f(\mathbf{x}) \psi(\mathbf{x}) d \Omega \quad \forall \psi \in H_{\partial \Omega_{D}}^{1}(\Omega),
$$

where $\psi(\mathbf{x})$ are test functions. After integrating by parts and using boundary conditions, we obtain:

$$
-\int_{\Omega} \nabla U(\mathbf{x}) \nabla \psi(\mathbf{x}) d \Omega=\int_{\Omega} f(\mathbf{x}) \psi(\mathbf{x}) d \Omega .
$$

According to the IGA paradigm, the temperature field is represented using B-spline basis functions. For a 2D problem, we have:

$$
U(\mathbf{x})=\mathcal{T}(\xi, \eta)=\sum_{i=1}^{n_{i}} \sum_{j=1}^{n_{j}} \hat{N}_{i}^{p_{i}}(\xi) \hat{N}_{j}^{p_{j}}(\eta) T_{i j},
$$

where $\hat{N}_{i}$ functions are B-Spline basis functions and $\mathbf{u}=$ $(\xi, \eta) \in \mathcal{P}$ are domain parameters. Then, we define the test functions $\psi(\mathbf{x})$ in the physical domain such as:

$$
N_{i j}(\mathbf{x})=N_{i j}(x, y)=N_{i j}(\mathcal{T}(\xi, \eta))=\hat{N}_{i j}(\xi, \eta)=\hat{N}_{i}^{p_{i}}(\xi) \hat{N}_{j}^{p_{j}}(\eta) .
$$

The weak formulation Eq. (2) reads:

$$
\sum_{k=1}^{n_{k}} \sum_{l=1}^{n_{l}} T_{k l} \int_{\Omega} \nabla N_{k l}(\mathbf{x}) \nabla N_{i j}(\mathbf{x}) d \Omega=\int_{\Omega} f(\mathbf{x}) N_{i j}(\mathbf{x}) d \Omega .
$$

Finally, we obtain a linear system similar to that resulting from the classical finite-element methods, with a matrix and a right-hand side defined as:

$$
\begin{aligned}
M_{i j, k l} & =\int_{\Omega} \nabla N_{k l}(\mathbf{x}) \nabla N_{i j}(\mathbf{x}) d \Omega \\
& =\int_{\mathcal{P}} \nabla_{\mathbf{u}} \tilde{N}_{k l}(\mathbf{u}) B(\mathbf{u})^{T} B(\mathbf{u}) \nabla_{\mathbf{u}} \tilde{N}_{k l}(\mathbf{u}) J(\mathbf{u}) d \mathcal{P}
\end{aligned}
$$

where $J$ is the Jacobian of the transformation, $B^{T}$ is the transposed of the inverse of the Jacobian matrix. The above integrations are performed in the parameter space using classical Gauss quadrature rules.

Starting from a planar B-spline surface as computational domain, an isogeometric solver for thermal conduction problem (1) has been implemented in the $\mathrm{AXEL}^{1}$ platform, yielding a B-spline surface as solution field. Gauss-Seidel algorithm is employed to solve the linear system. Fig.1 shows an example of planar B-spline surface as computational domain and the corresponding isogeometric analysis results for two-dimensional heat conduction problems.

In order to improve the simulation results, refinement operation can be performed for two parametric directions. There are three kinds of refinement operations in isogeometric analysis: $h$-refinement by knot insertion, $p$-refinement by

\footnotetext{
${ }^{1}$ http://axel.inria.fr/
}

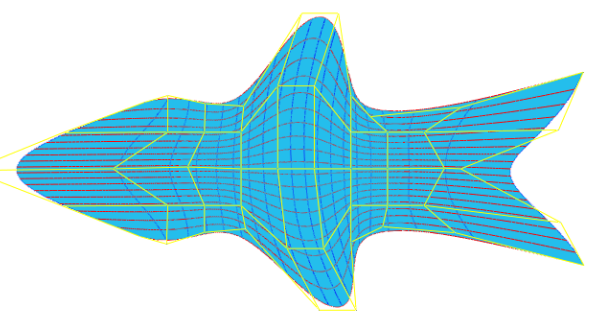

(a)

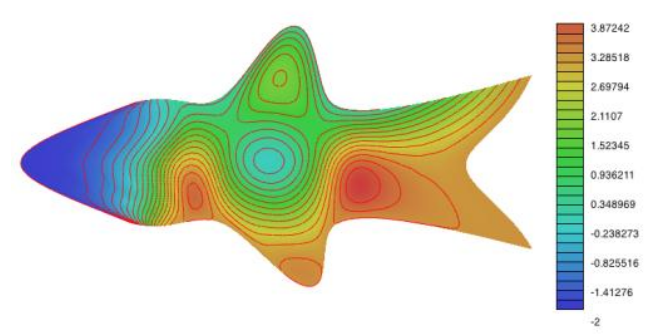

(b)

Fig.1 An example of isogeometric analysis for twodimensional heat conduction problem: (a) computational domain with control points and iso-parametric curves; (b) isogeometric simulation result.

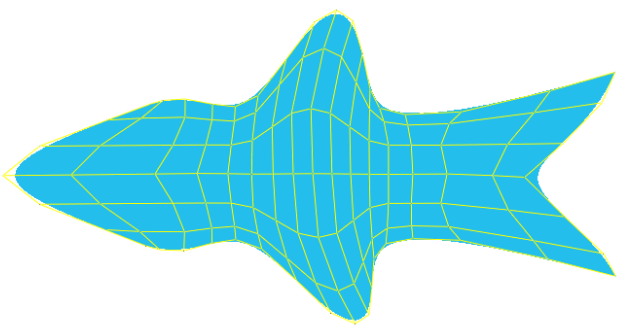

(a)

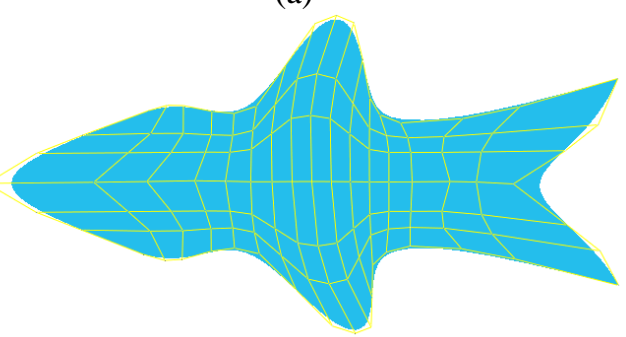

(b)

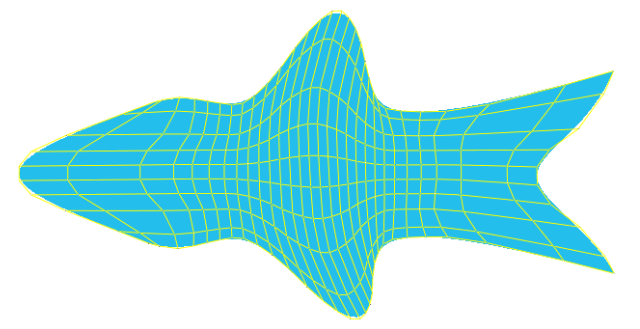

(c)

Fig. 2 Comparison of three kinds of refinement methods for the computational domain in Fig.1: (a) $h$-refinement; (b) $p$ refinement; (c) $k$-refinement. 
degree elevation operation, and $k$-refinement combining by knot insertion and degree elevation. The $k$-refinement operation is performed by elevating the degree of basis function to a desired order firstly followed by knot insertion thus obtaining the maximum available continuity. Compared with finite element analysis, the main advantage of refinement operations in isogeometric analysis is that the geometry of the computational domain can be kept while the degree of freedom increases. Fig. 2 presents an example to compare $h$ refinement, $p$-refinement and $k$-refinement in isogeometric analysis. Note that the number of control points increases during the refinement operation, and more degree of freedom can be achieved by $k$-refinement.

\section{ERROR ASSESSMENT METHOD BASED ON $k$-REFINEMENT}

Suppose that $U(\mathbf{x})$ is the exact solution, and $U_{h}(\mathbf{x})$ is the approximation solution obtained by isogeometric method in Section II, then the discrete error $e$ can be written as

$$
e=U-U_{h} \text {. }
$$

After performing Laplacian operator $\Delta$ on each side of (3), a posteriori error assessment can be obtained by resolving the following problem,

$$
\begin{array}{cc}
\Delta e=f-\Delta U_{h} & \text { in } \Omega \\
e=0 & \text { on } \partial \Omega
\end{array}
$$

From (4), the crucial point of a posteriori error estimation is the computation of $\Delta U_{h}(\mathbf{x})$. The following proposition is presented to show the computation of $\Delta U_{h}(\mathbf{x})$ directly on the parametric domain:

Proposition 1. Given B-spline parameterization $\sigma(\xi, \eta)=$ $(x(\xi, \eta), y(\xi, \eta))$ of the computational domain and the solution field $U_{h}(\mathbf{x})=U_{h}(x(\xi, \eta), y(\xi, \eta))=\mathcal{T}(\xi, \eta)$ over $\sigma(\xi, \eta)$, then $\Delta U_{h}(\mathbf{x})$ has the following form,

$$
\begin{aligned}
\Delta U_{h} & =\frac{\partial^{2} U_{h}}{\partial^{2} x}+\frac{\partial^{2} U_{h}}{\partial^{2} y} \\
& =\frac{J L_{\xi} \mathcal{T}_{\eta \eta}-J L_{\eta} \mathcal{T}_{\xi \xi}+L_{\xi \xi} \mathcal{T}_{\eta}-L_{\eta \eta} \mathcal{T}_{\xi}}{J K},
\end{aligned}
$$

where

$$
\begin{aligned}
J & =x_{\xi} y_{\eta}-x_{\eta} y_{\xi}, K=\left(x_{\xi} y_{\eta}\right)^{2}-\left(x_{\eta} y_{\xi}\right)^{2}, \\
L_{\xi} & =x_{\xi}^{2}-y_{\xi}^{2}, L_{\eta}=x_{\eta}^{2}-y_{\eta}^{2}, \\
L_{\xi \xi} & =\left(L_{\eta} y_{\xi \xi}-L_{\xi} y_{\eta \eta}\right) x_{\xi}-\left(L_{\eta} x_{\xi \xi}-L_{\xi} x_{\eta \eta}\right) y_{\xi}, \\
L_{\eta \eta} & =\left(L_{\eta} y_{\xi \xi}-L_{\xi} y_{\eta \eta}\right) x_{\eta}-\left(L_{\eta} x_{\xi \xi}-L_{\xi} x_{\eta \eta}\right) y_{\eta} .
\end{aligned}
$$

Proof. The idea of isogeometric analysis is to use the same mathematical representation for the computational domain and solution field. Suppose that the computational domain $\Omega$ is parameterized by the following planar B-spline surface:

$$
\sigma(\xi, \eta)=(x(\xi, \eta), y(\xi, \eta))=\sum_{i=1}^{n_{i}} \sum_{j=1}^{n_{j}} N_{i}^{d_{i}}(\xi) N_{j}^{d_{j}}(\eta) \boldsymbol{c}_{i j},
$$

In isogeometric analysis, the solution field of heat conduction problem (1) over the computational domain $\Omega$ has the following form,

$$
U_{h}(x(\xi, \eta), y(\xi, \eta))=\mathcal{T}(\xi, \eta)=\sum_{i=1}^{n_{i}} \sum_{j=1}^{n_{j}} N_{i}^{d_{i}}(\xi) N_{j}^{d_{j}}(\eta) T_{i j},
$$

Here $T_{i j}$ are the unknown variables in isogeometric analysis to be solved from the boundary condition and Eq. (1).

From $U_{h}(x(\xi, \eta), y(\xi, \eta))=\mathcal{T}(\xi, \eta)$, we have

$$
\begin{aligned}
& \frac{\partial \mathcal{T}}{\partial \xi}=\frac{\partial U_{h}}{\partial x} \frac{\partial x}{\partial \xi}+\frac{\partial U_{h}}{\partial y} \frac{\partial y}{\partial \xi} \\
& \frac{\partial \mathcal{T}}{\partial \eta}=\frac{\partial U_{h}}{\partial x} \frac{\partial x}{\partial \eta}+\frac{\partial U_{h}}{\partial y} \frac{\partial y}{\partial \eta}
\end{aligned}
$$

Then we can obtain

$$
\begin{aligned}
& \frac{\partial U_{h}}{\partial x}=\left(\frac{\partial \mathcal{T}}{\partial \xi} y_{\eta}-\frac{\partial \mathcal{T}}{\partial \eta} y_{\xi}\right) / J, \\
& \frac{\partial U_{h}}{\partial y}=\left(\frac{\partial \mathcal{T}}{\partial \eta} x_{\xi}-\frac{\partial \mathcal{T}}{\partial \xi} x_{\eta}\right) / J
\end{aligned}
$$

where $J=x_{\xi} y_{\eta}-x_{\eta} y_{\xi}$.

Similarly,

$\frac{\partial^{2} \mathcal{T}}{\partial^{2} \xi}=\frac{\partial^{2} U_{h}}{\partial^{2} x}\left(\frac{\partial x}{\partial \xi}\right)^{2}+\frac{\partial U_{h}}{\partial x} \frac{\partial^{2} x}{\partial^{2} \xi}+\frac{\partial^{2} U_{h}}{\partial^{2} y}\left(\frac{\partial y}{\partial \xi}\right)^{2}+\frac{\partial U_{h}}{\partial y} \frac{\partial^{2} y}{\partial^{2} \xi}$, $\frac{\partial^{2} \mathcal{T}}{\partial^{2} \eta}=\frac{\partial^{2} U_{h}}{\partial^{2} x}\left(\frac{\partial x}{\partial \eta}\right)^{2}+\frac{\partial U_{h}}{\partial x} \frac{\partial^{2} x}{\partial^{2} \eta}+\frac{\partial^{2} U_{h}}{\partial^{2} y}\left(\frac{\partial y}{\partial \eta}\right)^{2}+\frac{\partial U_{h}}{\partial y} \frac{\partial^{2} y}{\partial^{2} \eta}$,

From above two equations, we have

$$
\begin{aligned}
& \frac{\partial^{2} U_{h}}{\partial^{2} x}= {\left[y_{\eta}^{2}\left(\mathcal{T}_{\xi \xi}-\frac{\partial U_{h}}{\partial x} x_{\xi \xi}-\frac{\partial U_{h}}{\partial y} y_{\xi \xi}\right)-\right.} \\
&\left.y_{\xi}^{2}\left(\mathcal{T}_{\eta \eta}-\frac{\partial U_{h}}{\partial x} x_{\eta \eta}-\frac{\partial U_{h}}{\partial y} y_{\eta \eta}\right)\right] / K, \\
& \frac{\partial^{2} U_{h}}{\partial^{2} y}=\left[x_{\xi}^{2}\left(\mathcal{T}_{\eta \eta}-\frac{\partial U_{h}}{\partial x} x_{\eta \eta}-\frac{\partial U_{h}}{\partial y} y_{\eta \eta}\right)-\right. \\
&\left.x_{\eta}^{2}\left(\mathcal{T}_{\xi \xi}-\frac{\partial U_{h}}{\partial x} x_{\xi \xi}-\frac{\partial U_{h}}{\partial y} y_{\xi \xi}\right)\right] / K,
\end{aligned}
$$

where $K=\left(x_{\xi} y_{\eta}\right)^{2}-\left(x_{\eta} y_{\xi}\right)^{2}$.

Hence, we can obtain 


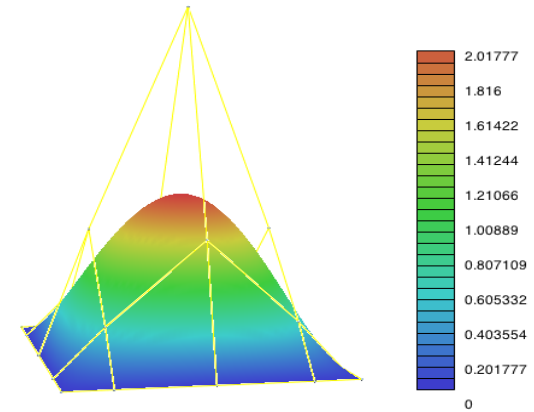

(a)

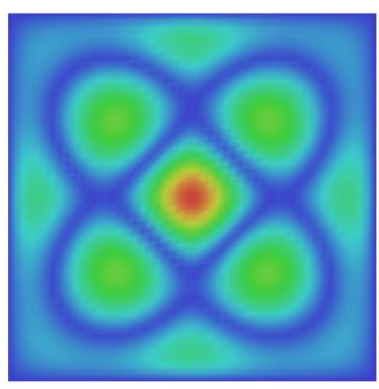

(d)

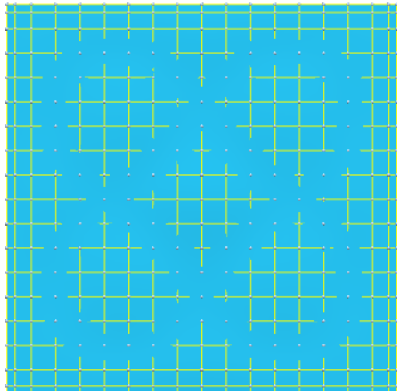

(b)

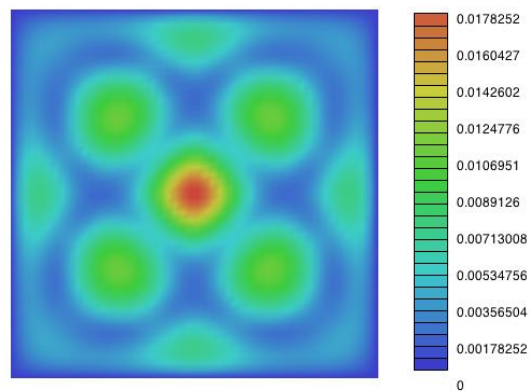

(e)

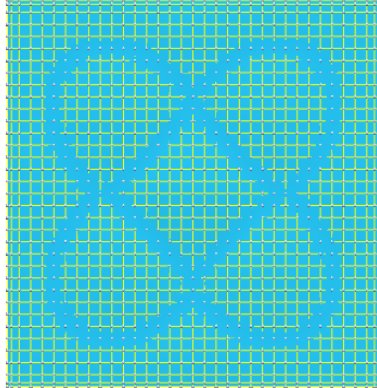

(c)

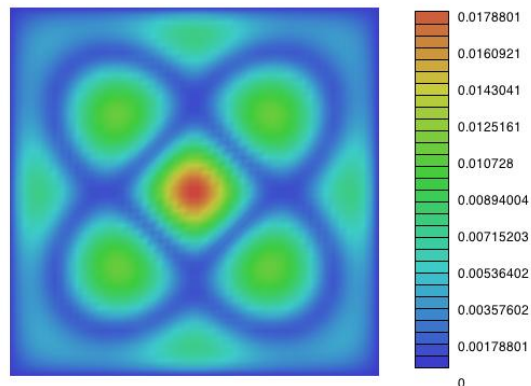

(f)

Fig.3 Comparison of error assessment method based on $h$-refinement and $k$-refinement: (a) isogoemetric solution surface with control points; (b) error surface obtained by three $h$-refinement steps; (c) error surface obtained by three $k$-refinement steps; (d) exact error color map; (e) color map of error surface in Fig. 3 (b); (f) colormap of error surface in Fig. 3 (c).

$$
\begin{aligned}
\Delta U_{h}= & \frac{\partial^{2} U_{h}}{\partial^{2} x}+\frac{\partial^{2} U_{h}}{\partial^{2} y} \\
= & {\left[\left(x_{\xi}^{2}-y_{\xi}^{2}\right)\left(\mathcal{T}_{\eta \eta}-\frac{\partial U_{h}}{\partial x} x_{\eta \eta}-\frac{\partial U_{h}}{\partial y} y_{\eta \eta}\right)-\right.} \\
& \left.\left(x_{\eta}^{2}-y_{\eta}^{2}\right)\left(\mathcal{T}_{\xi \xi}-\frac{\partial U_{h}}{\partial x} x_{\xi \xi}-\frac{\partial U_{h}}{\partial y} y_{\xi \xi}\right)\right] / K \\
= & \left(J L_{\xi} \mathcal{T}_{\eta \eta}-J L_{\eta} \mathcal{T}_{\xi \xi}+L_{\xi \xi} \mathcal{T}_{\eta}-L_{\eta \eta} \mathcal{T}_{\xi}\right) /(J K) .
\end{aligned}
$$

where

$$
\begin{gathered}
L_{\xi}=x_{\xi}^{2}-y_{\xi}^{2}, L_{\eta}=x_{\eta}^{2}-y_{\eta}^{2}, \\
L_{\xi \xi}=\left(L_{\eta} y_{\xi \xi}-L_{\xi} y_{\eta \eta}\right) x_{\xi}-\left(L_{\eta} x_{\xi \xi}-L_{\xi} x_{\eta \eta}\right) y_{\xi}, \\
L_{\eta \eta}=\left(L_{\eta} y_{\xi \xi}-L_{\xi} y_{\eta \eta}\right) x_{\eta}-\left(L_{\eta} x_{\xi \xi}-L_{\xi} x_{\eta \eta}\right) y_{\eta} .
\end{gathered}
$$

This completes the proof.

The approximation error surface $e$ from (4) also has a $\mathrm{B}$ spline form. Different from the method in [16], we perform several $k$-refinement operations to achieve more accurate results. Though it is much more expensive, we can use it as an error assessment method for isogeometric simulation solutions.

As a summary, the procedure of error assessment method for model problem (1) can be described as follows,
Input: the isogeometric solution $U_{h}(\mathbf{x})$ over computational domain $\Omega$

Output: the error surface $e$

1. Compute $\Delta U_{h}(\mathbf{x})$ according to Proposition 1;

2. Solve the isogeometric analysis problem (4) with several $k$-refinement steps ;

3. Output the error surface $e$.

\section{EXAMPLES AND COMPARISONS}

In this paper, we test the error assessment methods based on $h$-refinement and $k$-refinement for the heat conduction problem (1) with source term

$$
f(x, y)=-\frac{4 \pi^{2}}{9} \sin \left(\frac{\pi x}{3}\right) \sin \left(\frac{\pi y}{3}\right) .
$$

For this problem with boundary condition $U_{0}(x)=0$, the exact solution over the computational domain $[0,3] \times[0,3]$ is

$$
U(x, y)=2 \sin \left(\frac{\pi x}{3}\right) \sin \left(\frac{\pi y}{3}\right) .
$$

Fig. 3 illustrates an example over the computational domain $[0,3] \times[0,3]$, which has exact solution for problem (1). The isogeometric solution surface with control points are shown in Fig. 3(a), the error surface obtained by three $h$-refinement steps is illustrated in Fig. 3 (b), Fig. 3 (c) shows the error 


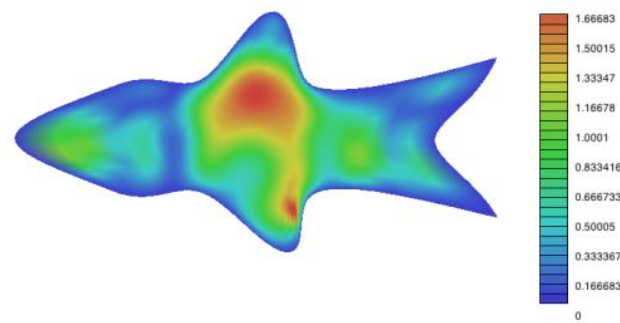

(a)

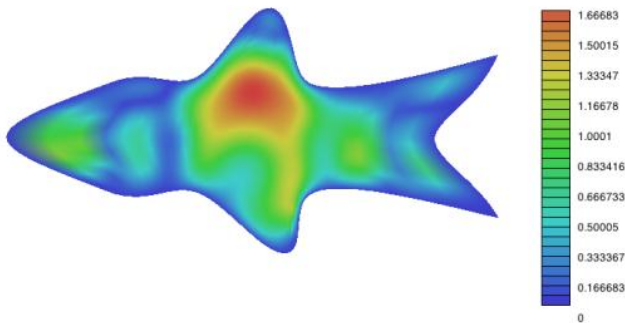

(b)

Fig.4 Error assessment for the simulation result in Fig. 1: (a) $h$-refinement; (b) $k$-refinement.

surface obtained by three $k$-refinement steps. The exact error color map for this example is shown in Fig. 3 (d), Fig. 3 (e) illustrate the color map of the error surface in Fig. 3(b), the color map of the error surface in 3(f) is shown in Fig. 3 (c) . From this example, we can find that $k$-refinement method can achieve a better approximation of the exact error surface than $h$-refinement method.

As an example with unknown exact solution, the error color map of simulation result in Fig. 1 obtained by $h$-refinement method and $k$-refinement method are shown in Fig. 4. Note that the original design of computational domain has significant impact on the simulation results. Hence, for different parameterization of computational domain, the resulted error surface obtained by the proposed method is also different.

\section{CONCLUSIONS}

A new error assessment method for isogeometric analysis of two-dimensional heat conduction problems is proposed in this paper. The basic idea is to resolve the isogeometric analysis problem with several $k$-refinement steps. The main feature of the proposed method is that the resulted error estimation surface also has a B-spline representation. It can be used as an error assessment method for isogeometric analysis results. The efficiency of the proposed method is proved by several comparison examples.

In the future, we will generalize the proposed method to $3 \mathrm{D}$ case and employ it to validate the $r$-refinement method in 3D isogeometric analysis as in [16].

\section{ACKNOWLEDGMENT}

The authors are partially supported by the 7th Framework Program of the European Union, project SCP8-218536 "EXCITING". The first author is partially supported by the National Nature Science Foundation of China (No.61004117), the Zhejiang Provincial Natural Science Foundation of China under Grant No.Y1090718, Defence Industrial Technology Development Program (No.A3920110002), and the Open Project Program of the State Key Lab of CAD \&CG (A1105), Zhejiang University.

\section{REFERENCES}

[1] M. Aigner, C. Heinrich, B. Jüttler, E. Pilgerstorfer, B. Simeon and A.V. Vuong. Swept volume parametrization for isogeometric analysis. In
E. Hancock and R. Martin (eds.), The Mathematics of Surfaces (MoS XIII 2009), LNCS vol. 5654, Springer, 19-44, 2009

[2] F. Auricchio, L.B. da Veiga, A. Buffa, C. Lovadina, A. Reali, and G. Sangalli. A fully locking-free isogeometric approach for plane linear elasticity problems: A stream function formulation. Computer Methods in Applied Mechanics and Engineering, 197:160-172, 2007.

[3] L. Beirao da Veiga, A. Buffa, J. Rivas and G. Sangalli. Some estimates for $h-p-k$-refinement in isogeometric analysis. Numerische Mathematik, 2011, 118(2): 271-305

[4] Y. Bazilevs, L. Beirao de Veiga, J.A. Cottrell, T.J.R. Hughes, and G Sangalli. Isogeometric analysis: approximation, stability and error estimates for $h$-refined meshes. Mathematical Models and Methods in Applied Sciences, 6:1031-1090, 2006.

[5] Y. Bazilevs, V.M. Calo, T.J.R. Hughes, and Y. Zhang. Isogeometric fluid structure interaction: Theory, algorithms, and computations. Computational Mechanics, 43:3-37, 2008.

[6] Y. Bazilevs, V.M. Calo, J.A. Cottrell, J. Evans, T.J.R. Hughes, S. Lipton, M.A. Scott, and T.W. Sederberg. Isogeometric analysis using T-Splines. Computer Methods in Applied Mechanics and Engineering, 199(5-8): 229-263, 2010.

[7] D. Burkhart, B. Hamann and G. Umlauf. Iso-geometric analysis based on Catmull-Clark subdivision solids. Computer Graphics Forum, 29(5): 1575-1584, 2010.

[8] J.A. Cottrell, T.J.R. Hughes, and A. Reali. Studies of refinement and continuity in isogeometric analysis. Computer Methods in Applied Mechanics and Engineering, 196:4160-4183, 2007

[9] J.A. Cottrell, A. Reali, Y. Bazilevs, and T.J.R. Hughes. Isogeometric analysis of structural vibrations. Computer Methods in Applied Mechanics and Engineering, 195:5257-5296, 2006.

[10] M. Dörfel, B. Jüttler, and B. Simeon. Adaptive isogeometric analysis by local h-refinement with T-splines. Computer Methods in Applied Mechanics and Engineering, 199(5-8): 264-275, 2010.

[11] H. Gomez, V.M. Calo, Y. Bazilevs, and T.J.R. Hughes. Isogeometric analysis of the Cahn-Hilliard phase-field model. Computer Methods in Applied Mechanics and Engineering, 197:4333-4352, 2008.

[12] T.J.R. Hughes, J.A. Cottrell, Y. Bazilevs. Isogeometric analysis: CAD, finite elements, NURBS, exact geometry, and mesh refinement. Computer Methods in Applied Mechanics and Engineering 194, 39-41: 4135-4195, 2005

[13] T. Nguyen, B. Jüttler. Using approximate implicitization for domain parameterization in isogeometric analysis. International Conference on Curves and Surfaces, Avignon, France, 2010.

[14] N. Nguyen-Thanh, H. Nguyen-Xuan, S.P.A. Bordasd and T. Rabczuk Isogeometric analysis using polynomial splines over hierarchical $\mathrm{T}$ meshes for two-dimensional elastic solids. Computer Methods in Applied Mechanics and Engineering, 2011, 21-22: 1892-1908

[15] G. Xu, B. Mourrain, R. Duvigneau, A. Galligo. Optimal analysis-aware parameterization of computational domain in isogeometric analysis. Proc. of Geometric Modeling and Processing (GMP 2010), 2010, 236254.

[16] G. Xu, B. Mourrain, R. Duvigneau, A. Galligo. Parameterization of computational domain in isogeometric analysis: methods and comparison.Computer Methods in Applied Mechanics and Engineering, 200(23-24): 2021-2031, 2011 